\title{
Secure Grid Computing with Trusted Resources and Internet Datamining
}

\author{
Kai Hwang \\ University of Southern California \\ Los Angeles, CA. 90089 USA
}

\begin{abstract}
Internet-based Grid computing is emerging as one of the most promising technologies that may change the world. Dr. Hwang and his research team at the University of Southern California (USC) are working on selfdefense tools to protect Grid resources from cyber attacks or malicious intrusions, automatically. This project builds an automated intrusion response and trust management system to facilitate authentication, authorization, and security binding in using metacomputing Grids or peer-to-peer web services. The trusted GridSec infrastructure supports Internet traffic datamining, encrypted tunneling, optimized resource allocations, network flood control and anomaly detection, etc. The USC team is developing a NetShield library to protect Grid resources. This new security system adjusts itself dynamically with changing threat patterns and network traffic conditions. This project promotes the acceptance of Grid computing through international collaborations with the research groups in INRIA, France, Chinese Academy of Sciences, and Melbourne University. The fortified Grid infrastructure will benefit securitysensitive allocations in digital government, electronic commerce, antiterrorism activities, and cyberspace crime control. The broader impacts of this ITR project are far reaching in an era of growing demand of Internet, Web and Grid services.
\end{abstract}

